\title{
LA MATERIA PRIMA COMO FUNDAMENTO DE LA NATURALEZA EN LA EDAD MEDIA (TRES LECTURAS DE LA HYLE ARISTOTÉLICA)
}

Antonio Pérez Estévez*

SINTESIS - La tradicional lectura de la materia prima aristotélica $(u \check{\lambda} \eta)$, que está próxima al ser pero accidentalmente es un no ser, la presenta, por un lado, como eterna y en potencia para recibir formas; por otro lado, como generable y corruptible, en cuanto constituyente de la sustancia primera. Averroes va a entender la materia prima como eterna y perteneciendo al ámbito del ser, pero con un modo de ser en potencia, esencialmente distinto del modo de ser en acto de la forma. Ser en potencia de la materia prima que la dispone para existir después en acto, es decir, la materia posee un ser en potencia incompleto que será completado por el ser en acto de la forma y constituir una sustancia individual. La materia prima de Tomás de Aquino, por otro lado, no pertenece, por sí misma, al ámbito del ser, que se reduce a ser en acto o ser formal. Es un no ente, un no existente que puede ser. Su pertenencia al ser la recibe del ser en acto de la forma en la sustancia corpórea. La imposibilidad, para la materia prima, de existir sin forma es absoluta porque considera que la proposición "la materia existe" encierra en sus términos contradicción. Finalmente, para Olivo, la materia prima está dotada de un acto de ser si bien distinto del acto de ser de la forma. Si el acto de ser de la forma es determinativo e indeterminable, el acto de ser de la materia prima es indeterminado y determinable. El acto de ser formal determina y perfecciona al acto de ser material para constituir una sustancia individual corpórea. - De las distintas maneras de concebir la hyle se seguirán distintas maneras de concebir la naturaleza.

PALABRAS-CLAVE - Materia prima. Naturaleza. Aristoteles. Tomás de Aquino. Pedro Olivi.
ABSTRACT - The traditional reading of the Aristotelian first matter that almost it is and accidentally it is not, it's presented, on one hand, as eternal and in power to receive forms; on the other hand, as generable and comuptible, as soon as constituent of the first substance. Averroes will understand the first matter as eternal and belonging to the environment of being, but with a way of being in power, essentially different from the way of being in act of the form. To be in power of the first matter that it prepares it to exist later in act, that is to say, the matter possesses a being in incomplete power that will be completed by the being in act of the form and to constitute an individual substance. The first matter of Thomas Aquinas, on the other hand, doesn't belong, for itself, to the being's environment that she extends only to the being in act or formal being. It is not a non entity, an non existent that can be. Its ownership to the being receives it from the being in act de la forma in the corporal substance. The impossibility, for the matter prima, of existing without form is absolute because it considers that the proposition "the matter exists" contains in its terms contradiction. Finally, for Olivo, the matter prima it is endowed with an act of being although different from the act of being de la forma. If the act of being of the form is determinative and indeterminable, the act of being of the first matter is uncertain and determinable. The formal act of being determines and perfects the act of being of material to constitute a corporal individual substance. From the different ways of conceiving the hyle different ways will be continued of conceiving the corporal substances and the nature.

KEY WORDS - First Matter. Nature. Aristotle. Aquinas. Peter Olivi.

* Professor na Universidade Zúlia/Venezuela.

\begin{tabular}{|l|l|l|l|l|l|}
\hline VERITAS & Porto Alegre & v. 44 & n. 3 & Setembro 1999 & p. 593-606 \\
\hline
\end{tabular}


La palabra naturaleza echa sus raíces etimológicas en la palabra latina natura la cual es derivada del verbo nascor, nasci, natus que significa nacer, originarse. De manera semejante física proviene de la palabra griega $\phi v \omega$ que significa igualmente nacer o engendrarse. La física o naturaleza era para los antiguos el entorno natural en el que las cosas nacían y perecían, se generaban y se corrompian. Era el ámbito del movimiento continuo en el que los seres aparecen y desaparecen, al que también pertenecía el ser humano. Ante este continuo hacerse de la naturale$\mathrm{za}$, los pensadores más antiguos reaccionaron de dos maneras distintas: o bien negaron la realidad del hacerse, del generarse y del corromperse, y lo redujeron a una pseudo realidad o apariencia (eleatas) o bien lo aceptaron como la única realidad pero sometida a una ley, a un logos que regulaba con su camino de subida y de bajada este continuo engendrarse y perecer (Heráclito).

La física o naturaleza tiene una relación profunda desde los inicios mismos de la cultura griega con la hýlê (ǔ $\lambda \eta$ ), bosque, madera o selva en la que el poder de la naturaleza se muestra en todo su esplendor. En latín la hyle se dice materia (madera) cuya raíz nos conduce a la palabra originaria mater madre o lo femenino origen de todo lo que se genera y se corrompe.

Aristóteles echa mano de las conocidas cuatro causas para explicar en su física este mundo natural que nace y perece. Pero cuando va a tratar de explicar el fundamento último del cambio, recurre a un substrato permanente que soporta los cambios de forma. Lo que cambia - nos referimos aquí al cambio profundo de una cosa en otra distinta o cambio sustancial - es, dice Aristóteles, la forma; pero, lo que permanece y soporta ese cambio de forma es la hyle o la materia. El cambio profundo de una cosa en otra, el continuo devenir, es una mezcla de ser y de no ser. El auténtico ser, siguiendo la tradición formalizante de Platón, se encuentra en la forma, en las Ideas luminosas. A la materia, por tanto, que es el soporte y fundamento de ese cambio, no le queda otro papel que el de no-ser. Veamos.

\subsection{La materia en Aristóteles}

Desde Aristóteles, el ser dice una relación esencial a la sustancia; de hecho, el ser se predica, en primer lugar y en sentido absoluto, de la sustancia. ${ }^{1}$ La sustancia encierra una consistencia en el ser que la hace ser-en-sí, existir en sí misma de una manera autónoma, mientras que el ser del accidente apenas si tiene consistencia pues su ser consiste precisamente en ser-en-otro. El ser del accidente es un ser parasitario que se alimenta y se sostiene siendo en una sustancia. Sustancia tiene aquí el significado propio de ser consistente o existir autónomo o separado; se dice, en primer lugar, de la sustancia primera o compuesta, del sýnolon ( $\sigma u ̉ v o \lambda o ̀ v$ ). Cuanto más duradera sea esa existencia autónoma más sustancia será. La sustancia natural o compuesta, el sýnolon ( $\sigma u ́ v o \lambda o v$ ), existe en el tiempo, es decir, se engendra y se corrompe, debido a que el no-ser de la materia la empuja a dejar de ser (lo que es) para convertirse en otra sustancia distinta; la

Aristóteles, Metafisica, $1028^{\mathrm{a}} 30$. 
sustancia compuesta de materia y forma es esencialmente una sustancia cuya consistencia está enraizada en el tiempo. ${ }^{2}$ Por eso, Aristóteles afirma que si existe alguna sustancia que sea forma pura, separable de la materia, su consistencia en el ser será eterna e inmutable y ha de ser de una manera excelente y divina. La ciencia de esa sustancia es una ciencia de lo divino, teología o filosofia primera

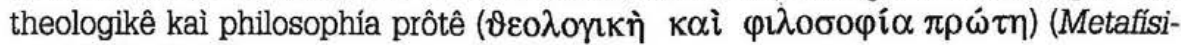
ca, 1026" 10-32).

Sustancia se dice, en consecuencia, de todo aquello que es autónomo. y consistente: se dice de la forma sustancial porque, según Aristóteles, es la causa formal y eficiente de toda consistencia en el ser de la sustancia (Metafísica 1041b8); se dice de la sustancia compuesta de materia y forma porque es la manera natural de ser, lo que implica ser en el tiempo. Y extrañamente, de alguna manera, $\pi \hat{\omega} \varsigma$, sustancia se dice también de la materia, aunque sea de una manera muy distinta a como se dice de la forma y del compuesto, debido a que la materia, en sí misma, está próxima al ser pero accidentalmente no-es (Física 192 $2^{\mathrm{a}}$ 3-5). Especifica Aristóteles que la sustancia se predica de la materia por el hecho de que es fundamento o soporte de todo cambio sustancial o generación (Física, 192 30). La materia, en cuanto fundamento de todo cambio o en su potencia o disposi-

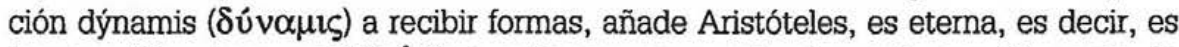
ingenerable e incomuptible. ${ }^{3}$ Pero, a la vez, en cuanto elemento constituyente de toda sustancia compuesta, de todo sýnolon ( $\sigma u ́ v o \lambda o v$ ), la materia es algo por la forma, ${ }^{4}$ lo que hace que, como parte del compuesto, esté enraizada en el tiempo. Es importante destacar este doble aspecto de la materia aristotélica que va a ser entendido de distintas formas por los distintos autores medievales: la materia, en cuanto fundamento o soporte de todo cambio, es eterna, es decir, ingenerable e incorruptible; la materia, en cuanto elemento constituyente del compuesto o sýnolon ( $\sigma u ́ v o \lambda o v$ ), es, igual que el ser sustancial, generable y corruptible, es decir, en el tiempo. Sustancia, según Aristóteles, se dice de la materia en su aspecto de ser fundamento de todo cambio o en su disposición o potencia insaciable de recibir nuevas formas.

Esta aparente contradicción de que la materia sea sustancia, cuando, en sí misma, no es consistente ni separada, y todo su ser actual lo recibe de la forma en la sustancia, trata de resolverlo Aristóteles, diciendo que el no-ser de la materia es casi ser o un no-ser por accidente, ouk òn eĩnai katà symbebêkòs tên kýlên (oúk

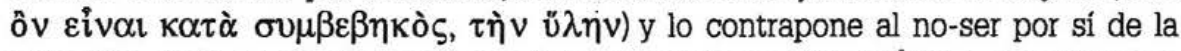
privación, de la que la sustancia en absoluto puede predicarse. ${ }^{5}$ De todas maneras, Aristóteles temina diciendo que la materia, debido al hecho de ser sustrato del cambio, tò hypokeímenon ( $\tau$ ò í

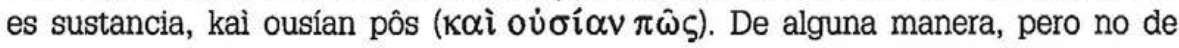
igual manera que la sustancia se dice del compuesto o de la forma, que poseen consistencia óntica y por sí mismas son.

Metafisica, $1039 \mathrm{~b} 24$ y $1042^{\mathrm{a}} 30$.

Aristóteles, Fisica, 192

Metafisica, $1041 \mathrm{~b}, 8-9$.

Aristóteles, Fisica,192 ${ }^{\mathrm{a}}, 5$. 
La materia, en cuanto sustrato de todo cambio sustancial, tiene una relación a las formas hacia las que está en potencia o en disposición de recibir. Aristóteles dice claramente que la materia es el compuesto individual pero en potencia, mientras que la forma es ese compuesto individual en acto. La relación del bronce a la estatua, de la madera a la cama es semejante a la que la materia amorfa (antes de recibir la forma) tiene a la forma. Análoga es la relación que tiene la materia a la sustancia, al individuo y al ser. Y es, precisamente por esa analogía, que la materia, en cuanto sustrato del cambio sustancial, es cognoscible (Física, 191 ${ }^{\text {a }}$ 511). Esta relación de la materia amorfa a la forma, es descrita también como un deseo o una tendencia semejante al que lo femenino siente por lo masculino y lo feo siente por lo bello, aunque la materia sea sólo accidentalmente femenina y fea (Física, 192 ${ }^{\mathrm{a}}$ 22-24). Existe, en consecuencia, en la materia amorfa aristotélica una relación a la forma.

\section{Tres lecturas de la Hyle aristotélica}

\subsection{La materia en Averroes}

Comencemos por decir que, para Averroes, la materia pertenece al ámbito del ser, si bien el ser de la materia sea un ser en potencia que lo hace deficiente e incapaz de existir de una manera autónoma.

"El ser se divide en ser en potencia y ser en acto [...]. En filosofía, la palabra potencia se refiere al modo de ser de una cosa que está en disposición de existir después en acto, tal como se predica de la materia."

El ámbito del ser se divide, según Averroes, en dos modos distintos de ser: ser-en-potencia propio de la materia y ser-en-acto propio de la forma. La materia, en consecuencia, es y se opone, en ese sentido, a la nada. Pero el ser de la materia es ser en potencia y, en este sentido, se opone al ser-en-acto de la forma. El modo de ser en potencia de la materia significa que está en disposición de existir después en acto. El modo de ser en potencia de la materia es un modo de ser imperfecto e incompleto; por eso, está en disposición de existir después en acto o de una manera completa y autónoma.

En segundo lugar, Averroes dice de la materia con su ser en potencia que es sustancia, aunque de un modo menos perfecto que lo es el compuesto y la forma:

"que la sustancia es más noble que el accidente [...] aquellas cosas de las que se dice la sustancia son de tres modos: uno de ellos es la materia prima que por si no es formada ni por sí es algo en acto [...]; el segundo modo es la forma por la que un individuo se hace un esto; el tercero, aquello que se hace a partir de ambas".?

6 Averroes, Compendio de Metafisica, texto árabe y traducción de Carlos Quirós Rodríguez, Madrid, 1919 , p. 45-46.

7 Averois Cordubensis Commentarium Magum in Aristotelis de Anima Librum Secundum, Cambridge, Mass., p. 130: opinamur quod substantia est nobilior accidente [...] omnia de quibus dicitur substantia sunt tribus modis; quorum unus est ut sit materia prima, quae per se non est formata neque aliquid per se in actu [... $\}$ : secundus autem est forma per quam individuum fit hoc; tertius est illud quod fit ex istis ambobus. 
Saquemos de este texto tres conclusiones fundamentales: 1) la sustancia es más noble que el accidente. 2) la materia prima per se no es formada ni per se es algo en acto, pero es uno de los tres modos de ser sustancia. 3) Los otros dos modos de ser sustancia -que son más perfectos que el de la materia - son la forma y el compuesto sustancial de materia y forma.

Siguiendo estos principios, podemos diseñar una escala ontológica de los seres corpóreos según Averroes: en primer lugar, estaria la sustancial individual compuesta de materia y forma; en segundo lugar, la forma que es la causa del ser en acto de la sustancia compuesta; en tercer lugar, la materia que, aún siendo en potencia, es una sustancia y posee un modo propio de ser; finalmente, el accidente sin consistencia propia y cuyo ser consiste en ser-en-otro.

Definamos las características de esa sustancia que es la materia prima. Dice de ella en el Compendio de Metafísica (p. 195) que

"La materia es en sí misma eterna; no está dotada de forma, por lo cual no puede tener causa eficiente, ya que ésta da al paciente la forma precisamente. Sin embargo ha de tener necesariamente un fin, el cual no es otro que la forma, pues de lo contrario existiŕa lo que de suyo no puede existir."

Deducimos de este texto que la materia prima, que es sustancia, es en sí misma eterna, debido a que no puede tener causa eficiente o formal que la produzca, porque la causa eficiente da a quien la recibe la forma, que es lo esencialmente distinto de la materia. Pero tiene un fin, que es la forma, con la que existe y es en acto, pues de otra forma existina lo que no puede existir o ser en acto. La imposibilidad de que la materia exista sola o sea en acto es absoluta; lo afirma repetidas veces: "la materia no puede existir sino mediante la forma (pues si existiera sin forma, se daria el caso de existir lo que no existe)" (Compendio de Metafísica, p. 144). Pero esta materia prima, aún sin existir de una manera autónoma y completa o ser en acto, es etema porque no tiene causa eficiente que la produzca y es uno de los modos de ser sustancia. El "modo de ser en potencia" de la materia prima por el que es eterna, no tiene causa eficiente que la produzca pero es un modo de ser sustancia, esencialmente distinto del ser en acto de la forma.

El modo de ser en potencia de la materia prima la dispone para existir después en acto en la sustancia individual, compuesta de materia y de forma. Es el compuesto de materia y forma el que tiene la consistencia suficiente para existir de una manera autónoma y duradera, aunque temporalmente, y sea el modo más perfecto de ser sustancia.

La forma determina la materia y la convierte en algo esto, en una sustancia individual compuesta. La materia, sin embargo, conserva su ser potencial en el compuesto ya que el ser en acto de la sustancia compuesta proviene de la forma que la hace ser una. Si la materia fuera en acto en el compuesto, entonces no se produciría un uno o una sustancia individual sino un agregado o añadido de dos seres en acto. ${ }^{8}$ La razón de la unidad sustancial, para Averroes, se debe a que se

8 Averrois Commentarium Magnum in Aristotelis de Anima, librum secundum, p. 139: materia non est hoc nisi per formam. Et si materia et forma essent in composito existentes in actu, tunc compositum non diceretur unum nisi sicut dicitur in rebus quae sunt unum secundum contactum et ligamentum. Modo autem, quia materia non differt a forma in composito nisi potentia, et compositum non est in actu nisi per formam, tunc compositum non dicitur unum nisi quia sua forma est una. 
da un solo ser en acto, que proviene de la única forma sustancial y que es la causa de esa unidad de la que participa la materia, en cuanto elemento constituyente de la sustancia. La materia, por tanto, existe sólo en la sustancia individual compuesta unida a la forma, de la que recibe su determinación y se convierte en un esto.

En la sustancia averroísta, la materia prima parece ser de dos maneras: 1) por sí misma en su etemidad 'es en potencia'; 2) por ser parte de la sustancia individual compuesta 'es en acto', aunque este 'ser en acto' de la materia provenga del ser que la forma da al compuesto y, por tanto, temporal, generable y corruptible.

Pero la entidad de ese ser en potencia de la materia prima que no le permite ser de una manera autónoma, no es una entidad simple sino una entidad compleja. En efecto, la deficiente entidad de la materia prima de Averroes es una entidad compuesta de un sujeto o sustancia y de una relación a las formas que ha de recibir. La materia encierra, como ya hemos dicho, la posibilidad $0^{\circ}$ disposición múltiple de recibir distintas formas sustanciales. Pero la posibilidad múltiple de recibir formas no puede existir sino en un sujeto o sustancia que posea tal posibilidad, o como dice Averroes textualmente: "la posibilidad presupone una cosa en la que existe y es un sujeto que recibe la cosa posible [...] la cosa o sujeto de posibilidad que recibe la posibilidad es la materia". . La posibilidad múltiple existe en la materia prima como en un sujeto o sustancia, lo que significa que la posibilidad múltiple viene a ser una suerte de accidente de relación que vincula a la materia-sustancia con las formas que va a recibir. Así lo expresa Averroes en el siguiente texto:

"Quid est subjectum, etc.? Id est, et modus essentiae eius est, quod non est demonstratum in actu, sed est quasi medium inter non esse simpliciter et esse in actu. Et accidit substantiae eius ut sit in potentia omnes formae: non quod potentia eius est in substantia, ita quod sit pars definitionis: quamvis si potentia esset in substantia eius tunc esse eius destrueretur ablatione potentiae [...] et universaliter, si potentia esset in substantia eius tunc substantia eius corrumperetur apud generationem et esset in praedicamento ad aliquid, non in praedicamento substantiae. Ex his igitur patet quod subjectum est substantia non potentia, neque privatio; pars enim substantiae est substantia."

Vemos que la potencia o disposición múltiple de la materia prima a todas las formas no está en la materia sustancia como parte de su definición o de sus esencia, pues si perteneciese a la esencia de la materia sustancia, desapareceria la materia sustancia al desaparecer la potencia en la nueva sustancia. Por eso, es evidente que el sujeto o sustancia de la materia prima ni es su potencia ni su privación. La potencia múltiple de la materia prima es un accidente de relación a

9 Destructio destructionum philosophiae Algazalis in the version of Calo Calonymus, Editado por $\mathrm{H}$. Zedler, Milwaukee, 1961, p. 124-125: "omne innovatum est possibile ante innovationem eius; et possibilitas praessuponit rem in qua existit, et est subjectum recipiens rem possibilem [...]. Quod sit subjectum possibilitatis res recipiens possibilitatem, et est materia".

10 Averrois Commentarium in Aristotelem, in librum primum Physicorum, Edición Iuntina, 1550, t. 70, fol. 41r, E-F. 
las formas que va a recibir. La materia prima de Averroes entraña una entidad compuesta de un sujeto o sustancia que soporta una forma accidental de relación a las formas que va a recibir.

Por último, es preciso decir que la materia prima de Averroes está afectada también del accidente cantidad, pero de un modo potencial. Accidente de cantidad que se manifiesta en las tres dimensiones indeterminadas, que acompañan siempre a la materia prima y de las que no puede jamás desnudarse, como afirma textualmente: materia prima nunquam denudatur a dimensionibus

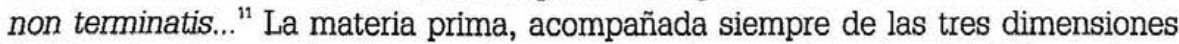
indeterminadas, es el sujeto o substrato de todo cambio sustancial. Dimensiones indeterminadas que se convierten en determinadas y concretas, en estas dimensiones con tales y tales límites, cuando la materia prima recibe la forma sustancial por la que se constituye una sustancia individual compuesta. ${ }^{12}$ Sin la dimensiones indeterminadas de la materia prima, no podría explicarse el origen del accidente cantidad y de la extensión en las sustancias corpóreas; lo extenso vendria de lo inextenso, lo que, según Averroes, es incomprensible. Las tres dimensiones indeterminadas es la primera forma -accidental- que existe en la materia prima, tal como lo afirma el Filósofo Cordobés. Forma accidental de cantidad indeterminada que existe en la materia sustrato o sustancia previa a toda forma sustancial, por la que esa forma accidental de cantidad en potencia se convertirá en acto y las tres dimensiones se harán determinadas o estas dimensiones concretas.

\subsection{La materia prima en Tomás de Aquino}

Veamos la lectura que Tomás de Aquino hace de la materia arsitotélica.

Comencemos afirmando que el ámbito del ser queda reducido al ser-en-acto, ens sumitur $a b$ actu essendi, ${ }^{13}$ ahora bien, ser en acto significa precisamente tener forma, porque la forma es aquello por lo que toda cosa es en acto, nam per formam unumquodque est actu, o, como dice en otro texto, es lo mismo ser acto que forma, ipsum autem quod est actus est forma. ${ }^{14}$ Todo ser tiene su origen en la forma o, como dice textualmente, el ser sigue siempre a la forma, sic esse consequitur formam, ${ }^{15} \mathrm{y}$, por tanto, sin forma, nada es. En consecuencia, la materia

1 De Substantia Orbis.(Aristotelis Opera cum Averrois Commentariis), vol. IX, edición Iuntina, 152-74, cap. I, 4B: et quia invenit omnes formas communicari in dimensionibus non terminatis, scivit quod prima materia nunquam denudatur a dimensionibus non terminatis, quia, si denudaretur, tunc corpus esset ex non corpore et dimensio ex non dimensione. También unas lineas después, cap. I, 4D: dimensiones igitur simpliciter, quae appelantur corpus simpliciter, non denudantur a materia prima...

Compendio de Metafísica, p. 121-122.

14 Questiones disputatae. De Veritate, Marietti, Turín-Roma, 1964, q.1, a.1 c.

In II de Anima, Marietti, 1956, No. 319-320: Illud est causa alicuius ut substantia, id est ut forma, quod est causa essendi. Nam per formam unumquodque est actu. Summa Theologica, Edición leonina, Ia., q.66, a. 1c: ipsum autem quod est actus, est forma, Dicere igitur materiam praecedere sine forma, est dicere ens actu sine actu, quod implicat contradictionem. Quaestiones disputatae. De Potentia, a. 21 c: forma dicitur esse vel ens, quia ea aliquid est...

Quaestiones disputatae. De anima, q. Unica, a. 6, c. 
prima queda excluida del ámbito del ser. Es más, Tomás de Aquino llega a afirmar que el ser, que es ser en acto, repugna a la razón de la materia que es en potencia o que la materia que es un no ente repugna a la razón de ente. ${ }^{16}$

La materia prima, en sí misma sin forma alguna, queda reducida ontológicamente a algo que no es ente sino en potencia, materia sicut non est ens nisi in potentia (Summa Theologica, Ia, q.5, a.3,ad 3um), a un no-ente que participa del bien porque a él tiende pero que no participa del ente. ${ }^{17}$ La materia prima, en sí misma, no participa del ser ni pertenece al ámbito del ser, es un no ente o un no existente que limita con la nada. Es por la forma que la materia prima entra en la esfera del ser y de él participa. ${ }^{18}$ Pero este no ente o no existente que es la materia no equivale al absoluto no ser o la nada. Tomás de Aquino entiende por no ente 0 no existente aquél ser que es solo en potencia y no en acto. ${ }^{19}$

Enntendida como un no ente, difícilmente el concepto de sustancia puede predicarse de la materia prima. De hecho, la consistencia en el ser de la materia tomista, que es un no ente o un ente en potencia, parece ser menor que la consistencia óntica del accidente, pues mientras el accidente es forma y acto - accidental pero forma y acto - y es en un sujeto sin que el sujeto sea su ser, el ser o acto de la materia, por el contrario, es la forma, forma est ipse actus materiae, y la esencia de la materia es lo contrario de la forma: la potencia o el no-ser. De aquí que sea posible, de una manera milagrosa, que el accidente exista sin sujeto que lo soporte; es, sin embargo, absolutamente imposible que la materia que es un noente, sea en acto $\sin$ forma ${ }^{20} \mathrm{La}$ escala ontológica tomista desciende por escalones de la siguiente manera: sustancia compuesta, forma, accidente y materia. La materia es el último escalón óntico, no participa del ser, es un no-ente que limita ya con la nada. Por esto, Tomás de Aquino repetirá que de la materia no puede predicarse propiamente la sustancia o el quod est, debido a que la materia no subsiste en el ser ya que ni es separable ni es esto o indivíduo. ${ }^{21}$ Admite que sustancia se predique de la materia solo denominative o concretive pero no per essentiam lo que significa que sustancia se diga de la materia de manera semejante a como el accidente se dice de la sustancia. Predicar sustancia denominative de la materia viene a significar que son esencialmente distintos, es decir, que mientras la sustancia es y es acto, la materia no-es y es sólo potencia. La materia es sustancia, sólo en la medida que participa del ser sustancial recibido de la forma. ${ }^{22}$

16

Quaestiones Quodlibetales. Quodlibet III, Marietti, Turín, 1956, q. 1, a. 1c: repugnat autem rationi entis non ens simul et secundum idem existens... et de huiumodi est materiam esse in actu sine forma.... esse actu repugnat rationi materiae.

17 Summa Theologica, Ia., q.5, a.2, ad 1um: materia prima participat bonum cum appetat ipsum... non autem participat ens, cum ponatur non ens.

18 Quaestiones disputatae. De Anima, q. Unica, a. 6 c: nam materia ex hoc quod recipit formam, participat esse... et licet materia non pertingat ad esse nisi per formam...

19

Summa Theol., Ia., q. 5, a. 2, ad 2um: per non existentia intelligamus, non ea simpliciter quae penitus non sunt, sed ea quae sunt in potentia et non in actu.

20 Quodlibetum Tertium, q. 1, a. 1, in contrarium: Quia Deus potest producere omnes actus secundarum causarum absque ipsis causis secundis, potest conservare in esse accidens sine subjecto. Sed materia secundum suum esse actuale dependet a forma in quantum forma est ipse actus eius; unde non est simile.

21 In 12 Libros Metaphysicorum, Marietti, 1964, No. 1289-1292.

22 Con respecto a la materia prima en Tomás de Aquino, ver: Pérez Estévez, Antonio, La Materia, de Avicena a la Escuela Franciscana, Ediluz, Maracaibo, Venezuela, 1998, cap. V, p. 133-162. 
La materia aristotélica que, en cuanto sustrato de todo cambio, era eterna, es decir, ingenerable e incorruptible ha quedado reducida, en Tomás de Aquino, a un no-ente que puede ser. Esa minima entidad que encierra la materia prima tomista, rayana con la nada, es reducida a pura potencia para recibir formas 0 , como dice textualmente, "la potencia de la materia no es otra cosa que su esencia". ${ }^{23}$ Pero la potencia o disposición de la materia para recibir formas expresa la relación de la materia con dichas formas y, en consecuencia, la materia tormista quedaría reducida a un no-ente que es potencia o relación a las formas que ha de recibir.

El ser etemo de la materia aristotélica ha desaparecido en Tomás de Aquino y todo el ser de la materia prima proviene de la forma. Esta concepción tomista de la materia encierra grandes dificultades que pueden resumirse en las siguientes preguntas: ¿Cómo el ser en acto de la forma puede dar el ser a algo esencialmente distinto como es la materia? ¿Cómo la materia prima puede ser el sustrato del cambio sustancial si su ser depende de la forma sustancial y, por tanto, se genera y se corrompe con ella? Reducir la materia prima a su poder ser o potencia para recibir formas, ¿no entraña reducirla a una simple relación, lo que haría difícil entender cómo una relación, o un accidente, puede ser sustrato de todo cambio sustancial? ¿No significa la exclusión de la materia del ámbito del ser, una amenaza de caer en un monismo formalizante?.

\subsection{La materia prima en Pedro de Juan Olivo}

La materia prima, en razón de su esencia, posee algún acto de ser, distinto del acto de ser de la forma. Acto de ser que significa algo real positivo o alguna entidad real distinta de la entidad real de la forma. ${ }^{24} Y$ este acto de ser es propio de la materia en razón de poseer una esencia propia, ya que la esencia, para Olivo, es acto 0 actualidad de ente.$^{25}$ La esencia es la raíz última de toda realidad; por eso, no puede hablarse de esencias no existentes o esencias en potencia de existir, como quiere Tomás de Aquino, pues, en ese caso, se hablaría de nada.

La sustancia, en Olivo, tiene dos actos de ser: el acto de ser propio de la materia y el acto de ser de la forma. Son actos de ser distintos y complementarios. El acto de ser de la materia es indeterminado y determinable o informable, mientras que el acto de ser de la forma es determinativo e indeterminable. ${ }^{26} \mathrm{El}$ acto de ser formal es determinativo, es decir, tiene la capacidad de determinar y especificar el acto indeterminado y determinable de la materia: El acto de ser material es imperfecto e incompleto y ha de ser perfeccionado y completado por el acto de ser de la forma para, juntos, constituir la sustancia individual compuesta. La materia,

Summa Theol., 12, q. 77, a. 1, ad 2um: Et ideo potentia materiae non est aliud quam eius essentia. Quaestiones in Secundum Librum Sententiarum, edición de Bemardo Jansen, Quarachi, 1922, q.XVI, p. 305-306.

Op. cit., p. 306: essentia est enim actus seu actualitas entis, non partialis sicut forma, sed potius totalis, quoniam ipsa est proprie illud per quod ens est ens.

Op. cit., q. XVI, pp. 309-310: Forma enim non est actus qualiscumque, sed solum actus determinativus et indeterminabilis; et quia huiusmodi actus nullam habet in se potentialitatem, sicut habet actus materiae... actus materialis distinguitur a formali, quia scilicet iste (materialis) est possibilis et informabilis, ille autem nullo modo. Non enim est inconveniens, si de se habeat esse indeterminatum et potentiale, sicut et habet actualitatem. 
en consecuencia, existente en la sustancia individual está dotada de dos actos de ser: el acto de ser propio, indeterminado e incompleto y el acto de ser determinado y completo de la forma., del que participa por ser principio constituyente de la sustancia individual. El acto de ser de la materia por indeterminado e incompleto no puede existir de una manera autónoma. Sólo perfeccionado y determinado por el ser en acto de la forma, puede existir de una manera autónoma en la sustancia individual.

La materia - igualmente que la forma y el compuesto - de alguna manera es sustancia porque es la raíz y fundamento primario de todo ente. ${ }^{27}$ Por sustancia, explica Olivo, "la materia no es solo potencia sino, además, algo sólido que tiene en sí la razón no sólo de una potencia sino de muchas ${ }^{n 28}$ La materia es una sustancia o algo sólido con un modo de ser absoluto que posee una relación múltiple a las formas que ha de recibir.

Vemos que la materia prima de Olivo, con un ser en acto propio y con una solidez absoluta, posee una potencia o relación a las formas. La entidad de la materia de Olivo es compleja. Es una sustancia que posee una potencia o relación múltiple a las formas o, como expresa textualmente recordando a Averroes, es "una secundum subjectum et plures secundum habilitates, hoc est, secundum modos se habendi quibus se habet per modum possibilis ad plures formas recipiendas" ${ }^{29}$ La materia de Olivo, que existe sólo en las sustanciales individuales, es, a la vez, sustancia sólida y potencia o disposición múltiple a las formas. La distinción entre ambos elementos - sustancia y potencia múltiple - la expresa Olivo por medio de sus típicas razones reales: la razón de esencia en la materia prima se distingue de la razón o razones de las potencias a las formas, aunque ambas razones pertenezcan a una y única realidad que es la materia. Igual que en Averroes, la materia prima de Olivo es, a la vez, una y múltiple, absoluta y relativa, si bien Olivo señalará que la cara absoluta de la materia se distingue de la cara relativa por tener razones reales distintas. ${ }^{30}$

Olivo sostiene que la materia prima, además de la composición de una cara absoluta y otra relativa, posee una extensión indeterminada, es decir, diversas partes extra partes que provienen de la esencia misma de la materia. ${ }^{31}$ Esta indeterminación de la extensión o del tamaño de la materia prima significa que entre las partes de la extensión no existe unión alguna ni división alguna, ni sitio o lugar alguno ni posición, aunque, de algún modo, están en capacidad de recibir el sitio y la extensión. ${ }^{32}$ La posición, el lugar, la división y la unión entre las diversas par-

27 Op. cit., p. 307: omnes philosophi velint eam esse partem constitutivam substantiarum habentium materiam et esse aliquo modo substantiam - unde dicunt substantiam dici analogice de materia et forma et composito - et velint eam esse radicem et fundamentum primarium totius entis...

28 Op. cit., p. 307: tenendum est quod materia non sit solum potentia, sed praeter hoc quiddam solidum habens in se rationem non solum unius potentiae sed etiam plurium.

29

30 Op. cit., q. XVII, ad Ium, p. 361.

Ver un estudio más detallado sobre esta doble cara en Pérez Estévez, Antonio, La Materia, de Avicena a la Escuela Franciscana, Maracaibo, Ediluz, 1998, cap. IX, p. 281-332.

31 Quaestiones in Secundum Librum Sententiarum, q. XVI, p. 307: Velint eam habere partes diversas secundum essentiam et ita quod suam essentiam et suam essentialem diversitatem non habeant a forma, sed potius per se ipsas.

32

Op. cit., q. XVI, p. 314: et hoc clarius patet in mole seu materia corporali de qua certum est quod eius partes per ipsas nullam habent inter se unionem nec divisionem nec situm nec positionem. 
tes de la extensión de la materia provendrán de la forma sustancial correspondiente que determinará esta extensión indeterminada que la materia prima esencialmente posee. Las partes indeterminadas de la extensión no están unidas o divididas de ninguna manera, pero son unibles y divisibles de acuerdo con las distintas formas sustanciales que la materia prima vaya recibiendo. La extensión o el tamaño los posee la materia prima en su misma esencia de una manera potencial. Dicho en otra forma, la materia prima, en cuanto sustancia, posee el accidente cantidad de una manera indeterminada o potencial; y este accidente de cantidad será determinado y en acto, o sea, tendrá una extensión determinada y sitio o lugar concreto en el momento mismo de recibir la forma sustancial que determina y perfecciona a la materia.

Por último, hemos de recordar, de pasada, una característica típica de la materia prima de Olivo: su movilidad intrínseca, por medio de la que puede informarse y transformarse en formas y sustancias naturales, una vez activada esta movilidad por un agente externo. ${ }^{33}$ La materia prima de Olivo encierra desde el momento mismo de su creación una movilidad que, activada por un agente externo, la hace transmutable en formas naturales. Y las formas naturales no se encuentran en la materia prima como en alguna forma o esencia distinta de la materia sino en la esencia misma de la materia en cuanto informe, móvil y terminable. ${ }^{34}$

La Escuela franciscana tiene como referencia, para determinar la consistencia óntica de la materia prima, el ser en otro del accidente, considerado como la menor entidad posible. La materia, que es una sustancia y tiene un ser en sí, sólido y absoluto, ha de tener más esencia y ser que el accidente. De aquí que si establecemos una escala ontológica descendente, tendríamos: en primer lugar la sustancia individual compuesta de materia y forma; en segundo lugar la forma que perfecciona y completa el ser imperfecto de la materia y, juntas, constituyen la sustancia individual; en tercer lugar, la materia prima con un ser en acto propio distinto del ser de la forma y una entidad absoluta que la hace ser sustancia; por último, el accidente, con su ser en otro con el que existe en la materia prima y, a través de ella, en la sustancia.

\section{Conclusión}

De las tres lecturas que hemos analizado, podemos deducir que cada una de ellas tiene un concepto distinto de sustancia corpórea y, en consecuencia, de naturaleza, en cuanto conjunto de sustancias naturales o corpóreas.

La unidad sustancial en Averroes es el resultado de la unión de dos seres igualmente eternos que se entremezclan para completarse: del ser en potencia de la materia y del ser en acto de la forma. Pero la causa eficiente de esa unidad, proviene del único ser en acto que es el de la forma. La forma hace que la sustan-

33 Op. cit., q. XXXI, p. 552: ...potius ipsum compositum seu congregatum dicitur fieri per motum, hoc est dictu, non forma influitur seu imprimitur materiae sed potius materia per motum fit formata seu habens formam.

34

Op. cit., p. 553: Hoc modo sunt omnes formae et earum esse creatae in materia; unde sunt ibi non per aliam essentiam ab essentia materiae sed quia hoc est de essentia omnis informis et mobilis et terminabilis, in quantum talis, quod intra suam essentiam et rationem secundum quid et indistincte includatur ratio suae formationis et terminationis. 
cia sea esto o individua y, por tanto, que sea una. Lo dice expresamente, al afirmar, que el compuesto no se dice uno a no ser porque su forma es una, tunc compositum non dicitur unum nisi quia sua forma est una. La materia prima, que es una sustancia y soporta directamente los accidentes de relación a las formas y de cantidad indeterminada o en potencia, es la causa de que la consistencia de la sustancia compuesta sea temporal en razón de su absoluto ser potencial que la hace estar siempre insatisfecha con la forma corpórea que soporta y busca, por tanto, cambiarla sin cesar.

La sustancia natural o corpórea tomista, de otro lado, no parece ser el resultado de la unión de dos seres. Todo el ser y el ser tal, en consecuencia, toda la unidad que se dan en una sustancia compuesta, según Tomás de Aquino, derivan de la forma, que es el único principio que es y que es en acto. La materia, por sí misma, es un no ente, un no existente - su esencia se reduce a la relación con las múltiples formas que ha de recibir - que entra en la esfera del ser, sólo en la sustancia natural o compuesta y en la medida en que participa del ser sustancial por medio de la forma. No es difícil entrever en esta posición la amenaza de reducir la sustancia a sólo la forma y caer en un monismo formalizante, como bien lo criticará Duns Scoto ${ }^{35}$ Es difícil también entender cómo la materia prima puede ser fundamento y sujeto del cambio sustancial, si su escaso ser deriva del ser formal que nace y perece con el ser de la sustancia.

Por último, la sustancia de Olivo es el resultado de la unión de dos seres en acto: el ser en acto indeterminado de la materia y el ser en acto determinativo de la forma, que determina y especifica al ser en acto de la materia. Es también la forma la que hace que la sustancia sea una e individua. La materia, que es sustancia sólida con su ser en sí y soporta tanto la relación accidental a las formas como la extensión indeterminada o sin posición y lugar, es la causa de la existencia temporal de la sustancia compuesta corpórea en razón de su absoluta indeterminación la cual nunca es determinada definitivamente por forma alguna corpórea y busca siempre una nueva forma sustancial. La indeterminación absoluta de la materia prima de Olivo no la deja existir de una manera autónoma; precisa siempre del ser en acto determinativo y más perfecto de la forma que lo determine y lo complete para, juntos, existir como una sustancia individual. Es, por tanto, la razón de subordinación de la materia prima a la forma y la complementariedad de los dos seres en acto la causa de la unidad de la sustancia en Pedro de Juan Olivo.

La naturaleza, como consecuencia de la distinta concepción de la sustancia, será entendida también de distinta manera. La naturaleza de Tomás de Aquino es un conjunto de sustancias corpóreas lo que no es otra cosa que un conjunto de seres formales en los que participan, de una extraña manera, unos no entes o no existentes, es decir, las materias que son la razón de que las sustancias individuales sean en el tiempo y se conviertan en otras sustancias. Así entendida, en la naturaleza de Tomás de Aquino, la forma es la única protagonista de ser, de ser en acto y de ser tal, que dispone a la sustancia para recibir la existencia. La

${ }_{35}$ Reportata Parisiensia, edición Vives, Paris, 1893, liber II, dist. XII, q. 1, p. 7, No. 11: qui dicunt quod materia est ens in potentia objectiva, dicunt quod materia est nihil in se. 
materia, como no ente o no existente, desempeña, sin embargo, el importante papel de empujar a la sustancia a dejar de ser o a no ser (lo que es) para convertirse en algo distinto sustancialmente.

En Averroes la naturaleza es el conjunto de sustancias corpóreas que, a su vez, son el resultado del continuo proceso de entremezclarse dos modos de ser eternos: el modo de ser en potencia de la materia y el modo de ser en acto de la forma. Modos de ser que se necesitan y se complementan para existir de una manera autónoma en las sustancias corpóreas. El modo de ser en acto de la forma, completa y determina al ser en potencia de la materia prima. Pero el ser en potencia de la materia prima es tan absolutamente en potencia que jamás se realiza del todo con el ser en acto de la forma y, como consecuencia, esa unión sustancial se rompe para dar lugar a una nueva unión sustancial con una nueva forma. La materia prima aporta, además de la temporalidad, la posibilidad de que la sustancia sea extensa y exista en un espacio, aunque será la forma la que convertirá esa extensión en una extensión determinada con tales y tales dimensiones.

La naturaleza, en Olivo, como conjunto de sustancias corpóreas y compuestas es el resultado de la unión de dos seres en acto distintos: el ser en acto indeterminado de la materia prima y el ser en acto determinativo de la forma. La forma da a la materia la perfección de ser esto o individuo en la sustancia corpórea que existe de una manera autónoma; la materia da a la sustancia la temporalidad y la posibilidad indeterminada de ser extensa o de existir en un espacio indeterminado. La forma sustancial hará concreto ese espacio y hará que esa extensión indeterminada de la materia prima se convierta en la extensión determinada con estas dimensiones concretas.

Si nos remitimos a las categonias de derecha e izquierda aristotélica en función de la consistencia óntica de la materia, tal como las describió Emst Bloch en su Avicena y la Izquierda Aristotélica, tendremos que ubicar muy a la derecha a Tomás de Aquino, en el centro a Averroes y más a la izquierda a Olivo. ${ }^{36}$

Tomás de Aquino ocupará la derecha con su materia prima que es un no ente o un no existente que puede ser y que entra en el ser sólo en la medida en que participa del ser formal en la sustancia compuesta. Todo el ámbito del ser, incluyendo todo tipo de ser determinado y específico lo abarca la forma, sin que la materia posea el derecho de participar en ese ámbito, a no ser a través de la forma.

El centro lo ocupará Averroes: su materia prima es pero es en potencia y constituye la sustancia compuesta entrelazándose con el ser en acto de la forma. La materia prima igual que la forma es eterna, aunque su ser potencial la hace imperfecta e incapaz de existir sola y de una manera autónoma. La materia prima de Averroes no posee una entidad simple sino compleja y soporta dos accidentes: el de relación o disposición a las formas que ha de recibir y la cantidad o extensión indeterminada.

36 Bloch, Erns, Avicena y la Izquierda Aristotélica, Ciencia Nueva, Madrid, 1966. 
A la izquierda de Averroes, colocamos a Pedro de Juan Olivo con su materia prima dotada de un ser en acto indeterminado que la hace ser sustancia y soportar una relación a las formas y una extensión indeterminada. La indeterminación de su ser en acto exige ser determinada y perfeccionada por la forma para constituir la sustancia corpórea temporal. Incluso, la materia prima de Olivo posee una movilidad esencial que la hace transformable en otras formas naturales corpóreas, una vez que es activada por un agente externo. En el mundo corpóreo natural, la materia prima parece desempeñar una función más importante que la forma.

Para finalizar, quiero señalar la notoria influencia de la noción de materia prima de Averroes sobre la materia prima de la Escuela Franciscana y, concretamente, de Olivo. Olivo retoma del Cordobés la doctrina de que la materia prima pertenece al ámbito del ser y fortalece ese ser material hasta convertirlo en ser en acto, pero indeterminado. El ser de la materia prima en Averroes era un ser en potencia; el ser de la materia prima de Olivo es un ser en acto indeterminado. Además, la sustancialidad de la materia prima de Olivo, igual que la de Averroes, soporta, como algo distinto, la relación a las formas y la extensión indeterminada. Olivo irá, incluso, más lejos al proveer a la materia prima de una movilidad que la hace transformable en formas naturales bajo la acción de un agente externo. Más que Avicena, es Averroes la fuente de una Izquierda Aristotélica, que pasa por la Escuela Franciscana y, muy especialmente, por Pedro de Juan Olivo. 